\title{
ANSIEDADE ENTRE CRIANÇAS E SEUS RESPONSÁVEIS PERANTE O ATENDIMENTO ODONTOLÓGICO.
}

\section{ANXIETY AMONG CHILDREN AND THEIR COMPANIONS AND RELATIVES IN DENTAL TREATMENT.}

\author{
Henrique Alberto Cunha Mendes Ferreira* \\ Arlete Maria Gomes Oliveira**
}

\begin{abstract}
RESUMO
O objetivo da pesquisa foi comparar escores de ansiedade perante o tratamento odontológico entre crianças e acompanhantes. Amostra de 44 crianças atendidas na clínica de Odontopediatria da Faculdade São Leopoldo Mandic, de ambos os sexos, idades de 4 a 11 anos. Utilizou-se a Escala de Ansiedade Visual (FIS) para crianças, identificando a ansiedade inicial e final por meio de 5 carinhas variando de muito feliz a muito triste, avaliada na primeira e na última consulta, e o questionário com a Escala de Ansiedade Dental de Corah's (DAS) para os responsáveis composto por 4 questões fechadas. Realizou-se análise descritiva por meio de tabelas de distribuição de frequências, cálculos da média, desvio padrão, mediana, valor mínimo e máximo, análise de correlação de Sperman entre escores total e de cada questão da escala de ansiedade (DAS) dos pais e o FIS dos pacientes. A comparação entre escores do FIS dos pacientes na primeira e última consulta foi realizada pelo teste de Wilcoxon, considerando-se o nível de significância de 5\%. Foram classificados como não ansiosos $4,5 \%$ dos pais e $31,8 \%$ com exacerbado grau de ansiedade. Não houve correlação significativa $(p>0,05)$ no estado de ansiedade das crianças e ansiedade de seus pais. Houve diminuição significante $(p<0,05)$ no escore médio do FIS referente à ansiedade da criança na última consulta. Conclui-se que a ansiedade dos pais não interferiu no comportamento da criança no tratamento odontológico, e que uma boa comunicação e a proximidade entre dentista e paciente são importantes para diminuir o grau de ansiedade durante o tratamento.
\end{abstract}

Descritores: Ansiedade no Tratamento odontológico • Dentista • Criança.

\section{ABSTRACT}

The aim of this research was to compare grades of anxiety in front of dental treatment among children and companion relatives. Sample of 44 children attended on the Pediatric Dentistry Clinic of Faculty São Leopoldo Mandic, both genders with age among 4 and 11 years old. During this data collection we have been applied the Facial Image Scale (FIS) for children in order to identify the inicial ansiety of child, by application of 5 little faces with variation from very sad until very happy. This collection was applied on the first and the last dental care for the child. Regarding the relatives and responsible we have been applied a questionary with Dental Anxiety Scale of Corah's (DAS) including 4 closed questions. Conducted a descriptive analysis by application of Frequency Distribution Tables, mean calculation, standard deviation, median, minimum and maximum value, Spearman correlation analysis between total scores and each answer of the Dental anxiety scale (DAS ) of the companions and the children's FIS. The relation betwen patient's first and last FIS score was performed by the Wilcoxon test, with significance level of $5 \%$. Classified as not anxious $4.5 \%$ of parents and $31.8 \%$ with excessive degree of anxiety. There was no significant correlation ( $p>0.05$ ) in the children's state of anxiety and anxiety of their companions. There was a significant decrease $(p<0.05)$ in the average FIES regarding the child anxiety in the last visit. The anxiety of the parents did not influence the behavior of the child in the dental treatment, and good communication and proximity between dentist and patient is important to reduce the level of anxiety during treatment.

Descriptors: Dental Anxiety • Dentist • Child. 


\section{N T R O D UÇÃO}

A ansiedade tem sido definida como um estado psíquico com predominância de sentimentos ameaçadores, que podem ser produzidos por estímulos internos e/ ou externos, reais ou imaginários, em situações desagradáveis próximas de acontecer. A ansiedade não possui um objeto pontual, é como se o indivíduo ficasse num estado de desamparo, perdesse a direção, esboçando reações consideradas inadequadas, sem que tenha premeditado tal comportamento ${ }^{1}$.

O medo é definido como uma sensação persistente e irracional, que resulta na compulsão para evitar um objetivo ou uma situação específica, é a expressão da necessidade de dependência, e ocorre em diversas etapas do desenvolvimento infantil. A ansiedade é considerada normal até certo ponto, pois prepara o organismo para os eventos futuros, sendo uma reação natural a um estímulo, apresentando ao paciente uma resposta apropriada, variando de acordo com a sua intensidade ${ }^{2}$. Conforme o desenvolvimento da criança, os medos vão mudando, saindo do mundo abstrato para sentimentos negativos, situações de violência, dor física, entre outros ${ }^{3}$.

Estudos clínicos e levantamentos epidemiológicos denotam significante interesse quanto à prevalência da ansiedade e sua influência no trabalho realizado pelo cirurgião-dentista ${ }^{4}$. A ansiedade diante do tratamento odontológico é o sentimento despertado por situações relacionadas ao atendimento que causam apreensão, desconforto, criando expectativa negativa no paciente, e seus fatores etiológicos mais significantes para o medo e ansiedade odontológica infantil são atitudes e experiências negativas passadas pelas mães e suas opiniões sobre tratamentos odontológicos ${ }^{5}$.

No que diz respeito à etiologia da ansiedade pelo tratamento dentário, nota-se que experiências dentais negativas são relevantes, assim como fatores relacionados à personalidade ${ }^{6}$. Outro fator importante que pode agravar e gerar a ansiedade são as experiências dolorosas, que podem variar quantitativa e qualitativamen- te de criança para criança, pois cada um tem sua história individual, experiências odontológicas e contexto socioeconômico e cultural distintos ${ }^{7,8}$.

A procura pela compreensão da ansiedade perante o tratamento odontológico tem estimulado a expansão gradual de inúmeros instrumentos psicométricos que possibilitam a análise dessa forma específica de ansiedade, a definição da sua prevalência e impacto, seu diagnóstico e tratamento individualizado ${ }^{9}$.

É possível que indivíduos apresentem reações adversas em relação à Odontologia porque foram influenciados por histórias e situações pontuais, como opiniões negativas veiculadas nos meios de comunicação, indicando que a Odontologia pode estar vinculada a traumas provocados pelos cirurgiões-dentistas ${ }^{10}$. Um aspecto fortemente associado à ansiedade e ao medo diante do tratamento odontológico em crianças é o medo relatado por membros da família ${ }^{11}$. Muitos estudos têm avaliado a ansiedade e o comportamento infantil durante os procedimentos odontológicos concomitantemente com a ansiedade da mãe da criança, com o intuito de buscar associações entre o estado emocional de ambas ${ }^{12,13}$.

Pesquisas analisando a ansiedade têm sido realizadas por meio de técnicas projetivas, principalmente por meio de questionários e termômetro da dor (escolher entre várias cores), embora autores as considerem pouco úteis para avaliação da ansiedade das crianças, uma vez que necessitariam do paciente certa maturidade para entender o significado do que está sendo exposto a ele ${ }^{14}$.

As técnicas projetivas são consideradas formas de comunicação indireta, facilitadoras no trabalho com crianças e adolescentes, onde a comunicação verbal direta nem sempre se mostra suficiente para a obtenção do material necessário para a coleta de dados. Essas técnicas são, em geral, lúdicas e permitem o acesso a fantasias, desejos, impulsos, afetos, conflitos, ansiedades e defesas ${ }^{15}$.

Outros estudos têm mostrado que um bom instrumento para avaliarmos crianças é o desenho, que reflete aquilo que nós somos no momento presente, inte-
FERREIRA HACM

OLIVEIRA AMG

ANSIEDADE ENTRE CRIANÇAS E SEUS RESPONSÁVEIS PERANTE O ATENDIMENTO odONTOLÓgICO.

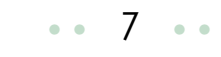

REV, ODONTOL.

UNIV, CID, SÃO PAULO

2016; 29(1): 6-17, JAN - ABR 
FERREIRA HACM :

OLIVEIRA AMG

ANS IEDADE ENTRE CRIANÇAS E SEUS RESPONSÁVEIS

PERANTE O

ATENDIMENTO ODONTOLÓGICO.

\section{8}

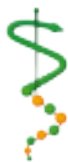

REV, ODONTOL. UNIV, CID, SÃO

PAULO

2017; 29(1): 6-17,

$J A N-A B R$ grando o passado e nossa história pessoal, é a manifestação do íntimo e suas reações subjetivas $^{16}$. Por volta dos dois anos de idade já são feitos os primeiros rabiscos, por puro prazer; aos poucos o pensamento passa a ser imaginativo quando a criança relaciona os rabiscos a elementos do seu meio; a partir de quatro anos o desenho é uma oportunidade para a criança organizar suas experiências, convertendo o pensamento em forma concreta ${ }^{17}$.

Em crianças de pouca idade, tem-se empregado a Escala Visual Analógica (EVA), que consiste em desenhos de faces expressando a "preocupação, neutralidade, e felicidade". A sua aplicação é fácil e rápida, podendo ser realizada tanto pelo dentista como pelo auxiliar, no momento que antecede ao tratamento, permitindo que o profissional elabore estratégias de abordagem adequadas ao paciente. A EVA pode ser utilizada também para avaliar o grau da dor que o paciente está sentindo durante o procedimento cirúrgico; para esse objetivo, o teste consiste numa linha reta com as seguintes identificações: sem dor, dor fraca, dor média, dor forte e dor insuportável. O paciente é instruído a marcar um ponto no local que melhor representa o grau de dor que está sentindo, em 3 momentos diferentes do procedimento cirúrgico (antes, durante e após) ${ }^{18}$.

$\mathrm{Na}$ busca de tentar elucidar os fatores causadores de ansiedade, que não são aqueles passados de pais para filhos, Ramos-Jorge et. al. ${ }^{12}$ apresentaram imagens positivas de tratamento dental às crianças a fim de prepará-las para o atendimento e verificaram, por meio do teste Venham Picture Test, o nível de ansiedade em três momentos distintos, comparando com o grupo-controle ao qual não eram mostradas as imagens. Os autores concluíram que a visualização de imagens de consultas odontológicas positivas não influenciou o nível de ansiedade da criança ${ }^{12}$.

Além da avaliação da ansiedade infantil prévia ao tratamento odontológico, o entendimento de outras causas desse estado emocional poderiam contribuir para maior sucesso no atendimento odontopediátrico, levando ao desenvolvimento de pesquisas sobre o tema, e outros fatores não dentais influenciadores e suas variá- veis têm sido exploradas nas pesquisas ${ }^{19}$. No que diz respeito à etiologia da ansiedade pelo tratamento dentário, nota-se que experiências dentais negativas são relevantes, assim como fatores relacionados à personalidade .

A experiência dolorosa pode variar quantitativa e qualitativamente de criança para criança, pois cada um tem sua história individual, experiências odontológicas e contexto socioeconômico e cultural distintos, fatores estes relevantes no agravo e causadores de ansiedade infantil ${ }^{8}$.

A imaturidade da idade também é um dos fatores que influencia o comportamento infantil no atendimento odontológico. Oliveira et. al. ${ }^{19}$, avaliando o comportamento infanto-juvenil na rotina de atendimento odontológico, verificaram que, diferentemente de crianças de menor idade, na faixa etária de 8 anos a criança tende a aumentar o seu círculo de amizades, desenvolvendo certo sentido de dever, aceitando normas e obrigações sociais, ampliando sua capacidade de raciocínio e compreensão, tornando mais fácil obter aceitação do tratamento odontológico.

Alguns autores afirmam que o êxito no atendimento odontológico infantil depende de alguns fatores como o estabelecimento de uma relação de confiança com a criança e o gerenciamento do comportamento de forma individualizada, e quando esses aspectos são levados em conta, a ansiedade infantil tende a diminuir $^{20}$. Técnicas de controle e condicionamento comportamental e psicológico na realização do atendimento ao paciente pediátrico são importantes, pois, independentemente do procedimento, nota-se que lidam com sentimentos como medo e ansiedade, sendo indispensável que se preste atenção à opinião dos pais a respeito das diversas opções de técnicas oferecidas, e dinamização do processo para que se diminua a ansiedade do paciente infantil por parte dos especialistas em odontopediatria e dos clínicos gerais ${ }^{21}$.

A observação do comportamento infantil auxilia o odontopediatra a utilizar as técnicas que viabilizam e facilitam o comportamento da criança a agir com parceria durante o tratamento odontológico, bem 
como a relação entre a ansiedade de pais e filhos também deve ser levada em conta, uma vez que o sucesso do tratamento odontopediátrico se deve à relação entre pais, filhos e profissional. Dessa forma esta pesquisa avaliou a influência entre a ansiedade de pares durante o atendimento odontológico.

\section{MATERIAIS E MÉTODOS}

Estudo epidemiológico, observacional, transversal de natureza quantitativa. Para a decisão do tamanho amostral, inicialmente contatou-se o setor de agendamento da clínica de graduação da Faculdade São Leopoldo Mandic para levantamento do número de pacientes agendados na clínica de odontopediatria em um semestre, sendo relatada uma estimativa de 40 agendamentos. Conforme informação, foram considerados, para tanto, todos os pacientes agendados $(n=40)$, atendidos no semestre. Esses cálculos estão embasados na literatura nos estudos citados $\mathrm{em}^{16,19,21,22}$.

A amostra contemplou ambos os gêneros, idade entre 4 e 11 anos, provenientes da clínica de Odontopediatria da Faculdade São Leopoldo Mandic e os respectivos acompanhantes dos que foram selecionados para tratamento em 2016. Foram incluídos os pacientes selecionados para atendimento na clínica de odontopediatria e que seja programados para mais de uma sessão para atendimento. Foram excluídas do estudo as crianças cujos pais se recusaram a assinar o Termo de Consentimento Livre Esclarecido (TCLE), e aquelas que não necessitaram de retorno. Após a seleção, a amostra final compôs-se de 44 pares (criança/pais), sendo 22 do sexo masculino e 22 do feminino.

Para a coleta dos dados foram utilizados Instrumentos que avaliaram a ansiedade da criança e dos responsáveis. O Facial Image Scale (FIS) ${ }^{23}$ é um Instrumento composto por desenho de cinco carinhas variando de muito feliz a muito triste. As crianças apontam a carinha que mais representa a sua condição no momento da consulta. O escore varia de 1 para a mais positiva, até 5 para a mais negativa. Algumas das vantagens dessa escala é que pode ser aplicada a crianças de pouca idade, é um método fácil e rápido, podendo ser aplicado até na sala de espera. A Escala de Ansiedade Dental de Corah Traduzida (DAS) ${ }^{24}$ consiste em um instrumento de boa confiabilidade para verificar o nível de ansiedade gerado pela situação de tratamento odontológico dos pais, procedendo-se à leitura e explicação de todas as questões para a escolha das respostas pelo entrevistado. Consiste em um teste psicométrico, em forma de questionário, que classifica os indivíduos em temerosos ou não em relação ao tratamento odontológico. É composta por quatro perguntas, com cinco alternativas de respostas para cada uma; para cada alternativa é atribuído um valor, em ordem crescente, numa escala de cinco pontos, e o escore total de pontos obtidos resulta do somatório das quatro questões. Esse instrumento é um questionário que apresenta quatro perguntas com cinco opções de resposta. $\mathrm{O}$ número de pontos pode variar de 4 para paciente não ansioso, até 20 para paciente muito ansioso.

Os questionários para avaliar a ansiedade dos pais eram na forma autoaplicáveis, e o pai ou acompanhante poderiam estar ou não no mesmo ambiente em que a criança se encontrava (clínica ou sala de espera). O pesquisador passou por um treinamento objetivando a adequada aplicação e conhecimento dos instrumentos (questionários) utilizados na pesquisa, verificando o tempo de duração da aplicabilidade, bem como possíveis falhas metodológicas.

Anteriormente foi realizado um estudo piloto, no qual foram examinados indivíduos selecionados aleatoriamente na sala de espera da clínica, com as mesmas características da amostra para a pesquisa, objetivando-se encontrar possíveis dificuldades para a realização da pesquisa e compreensão das perguntas do questionário pelos participantes, evitando-se dessa forma, interrupções durante o levantamento principal dos dados. Inicialmente foram selecionados os pacientes agendados para tratamento e seus acompanhantes, foi explicado o teor da pesquisa e assinado o TCLE. A criança era abordada no primeiro contato do paciente com o aluno de graduação, sendo aplicada a Escala
FERREIRA HACM

OLIVEIRA AMG

ANSIEDADE ENTRE CRIANÇAS E SEUS RESPONSÁVEIS PERANTE O ATENDIMENTO ODONTOLÓGICO.

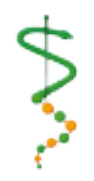

REV, ODONTOL.

UnIV. CID, SÃO

PAULO

2016; 29(1): 6-17, JAN - ABR 
FERREIRA HACM :

OLIVEIRA AMG

ANS IEDADE ENTRE CRIANÇAS E SEUS RESPONSÁVEIS

PERANTE O

ATENDIMENTO ODONTOLÓGICO.
$10 \ldots \vdots 5 \%$.

Este estudo foi submetido ao Comitê de Ética em pesquisa, da Faculdade São Leopoldo Mandic, recebendo aprovação (protocolo n ${ }^{\circ}$ CAAE: 54735216.6.0000.5374).

\section{RESULTADOS}

A amostra foi composta por 44 pares de voluntários (crianças e pais), sendo 22 do sexo feminino $(50,0 \%)$ e 22 do sexo masculino (50,0\%). A média de idade da amostra foi de 7,4 anos com $d p=2,0$, mínimo de 4 e máximo de 11 anos (Tabela 1). O escore médio do Facial Image Scale (FIS) na primeira consulta foi de 1,7 $(d p=0,9)$ e na última consulta foi de 1,3 $(\mathrm{dp}=0,5)$. Na Tabela 1 são apresentadas as estatísticas descritivas do escore de ansiedade dos pais pela Escala de Ansiedade Dental de Conrah Traduzida (DAS).

$\mathrm{Na}$ Tabela 2 é apresentada a distribuição de frequências dos pais de acordo com a escala DAS. Observa-se que apenas dois pais $(4,5 \%)$ foram classificados como não ansiosos. Do total de pais, 31,8\% foram classificados com Exacerbado Grau de Ansiedade.

Não houve correlação significativa ( $p>0,05)$ entre os escores de FIS dos pacientes e as questões do instrumento DAS dos pais nas quatro situações avaliadas, nas duas consultas (primeira e última) (gráficos 1 e 2).

Também não houve correlação significativa entre os escores do FIS dos pacientes nas duas consultas avaliadas e os escores total do DAS dos pais $(p>0,05)$ (Gráfico 3).

Tabela 1. Análise descritiva das variáveis estudadas.

\begin{tabular}{lll}
\hline \hline & Média (Desvio Padrão) & Mediana (Mínimo; Máximo) \\
\hline Idade & $7,4(2,0)$ & $7(4 ; 11)$ \\
$1^{\text {a }}$ Consulta & $1,7(0,9)$ & $1(1 ; 4)$ \\
Última Consulta & $1,3(0,5)$ & $1(1 ; 3)$ \\
Pais (ida ao dentista) & $2,7(1,1)$ & $2,5(1 ; 5)$ \\
Pais (sala de espera) & $2,4(1,4)$ & $2(1 ; 5)$ \\
Pais (turbina) & $2,8(1,2)$ & $3(1 ; 5)$ \\
Pais (limpeza) & $2,7(1,0)$ & $3(1 ; 5)$ \\
Pais (DAS) & $10,6(3,6)$ & $11(4 ; 18)$ \\
\hline \hline
\end{tabular}

Tabela 2. Distribuição de frequências da classificação dos pais pela escala de ansiedade DAS.

REV, ODONTOL.

UNIV, CID, SÃO

PAULO

$2017 ; 29(1): 6-17$

\begin{tabular}{lcc}
\hline \hline Grau de Ansiedade (DAS) & Frequência & Porcentagem \\
\hline Indivíduo Não Ansioso & 2 & 4,5 \\
Baixo Grau de Ansiedade & 12 & 27,3 \\
Moderado Grau de Ansiedade & 16 & 36,4 \\
Exacerbado Grau de Ansiedade & 14 & 31,8 \\
\hline \hline
\end{tabular}


Tabela 3. Comparação entre os escores de FIS dos pacientes na primeira e segunda consultas.

\begin{tabular}{lll}
\hline \hline & Média (Desvio Padrão) & Mediana (Mínimo; Máximo) \\
\hline $1{ }^{\text {a }}$ Consulta & $1,7(0,9)$ & $1(1 ; 4)$ \\
Última Consulta & $1,3(0,5)$ & $1(1 ; 3)$ \\
\hline \hline
\end{tabular}

p-valor $=0,0015$
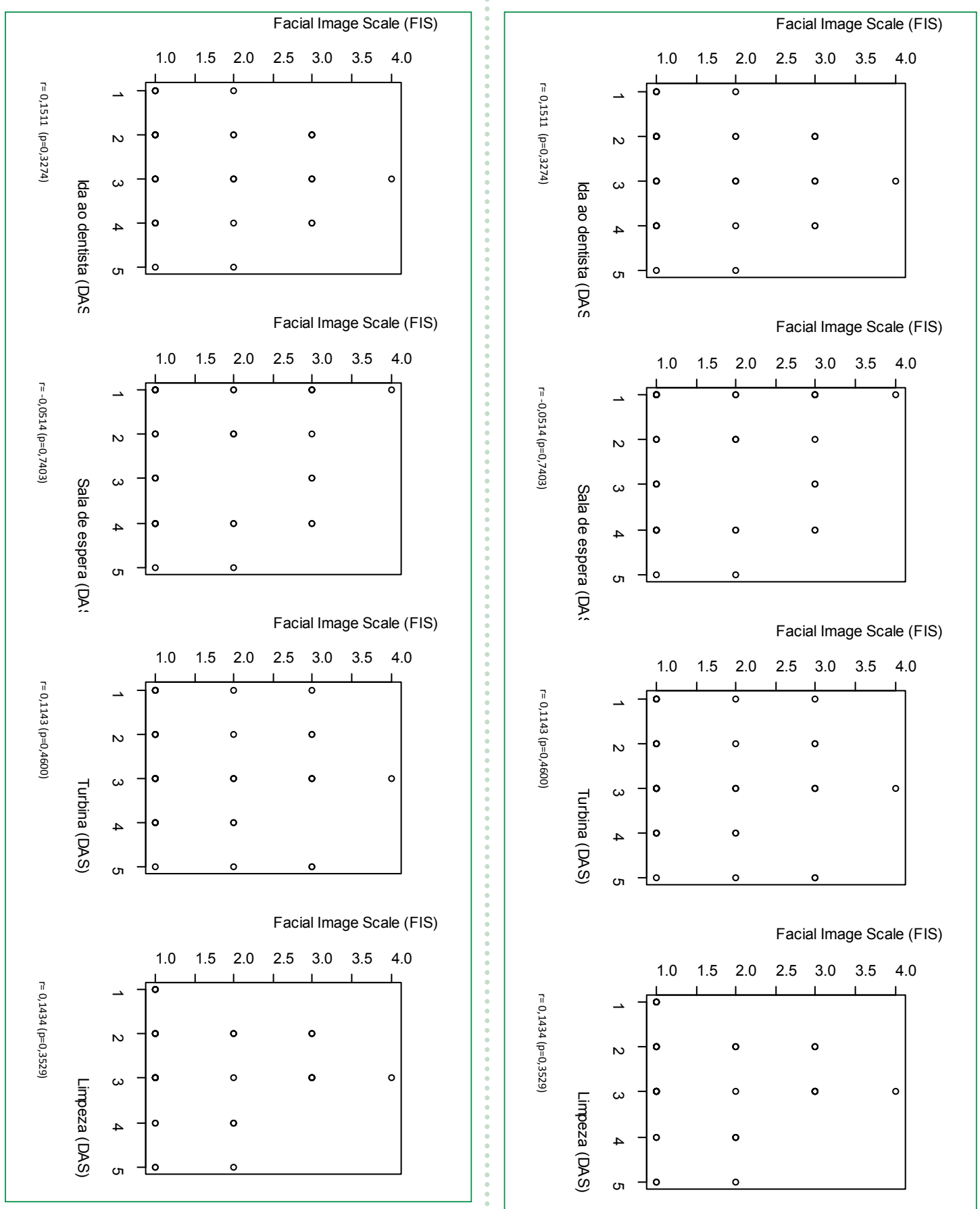

Gráfico 1. Análise de Correlação entre os escores FIS dos pacientes na primeira consulta e os escores das questões do DAS dos pais.

\footnotetext{
* Bioestat 5.0 statistical program (Mamirauá Maintainable Development Institute, Belém, Pará, Brazil, 2009).

** R Core Team (2015). R: A language and environment for statistical computing. R Foundation for Statistical Computing, Vienna, Austria. URL https://www.R-project.org/.
}

FERREIRA HACM OLIVEIRA AMG

ANSIEDADE ENTRE CRIANÇAS E SEUS RESPONSÁVEIS PERANTE O ATENDIMENTO ODONTOLÓGICO.

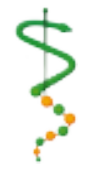




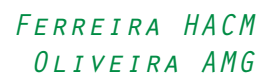

ANSIEDADE ENTRE CRIANÇAS E SEUS RESPONSÁVEIS

PERANTE 0 ATENDIMENTO ODONTOLÓGICO.

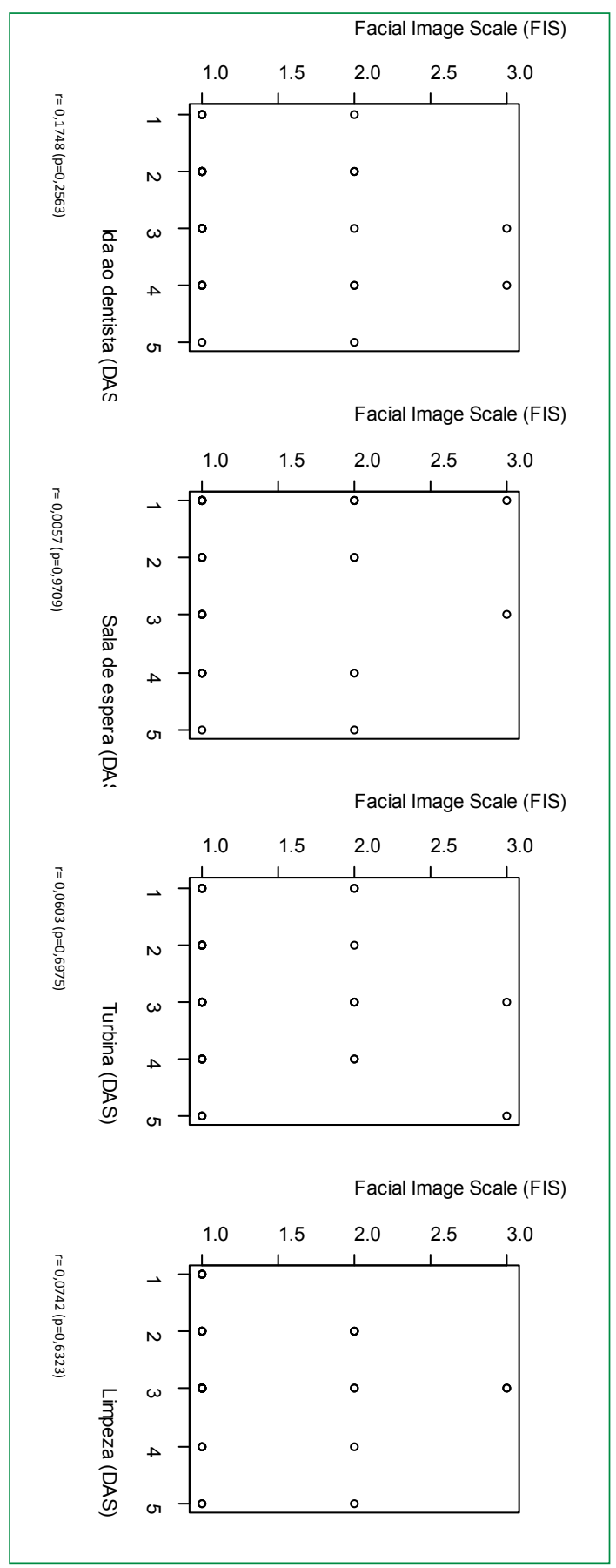

Gráfico 2. Análise de Correlação entre os escores FIS dos pacientes na última consulta e os escores das questões do DAS dos pais.

Os resultados para os escores do FIS dos pacientes entre a primeira e última consultas apresentaram diminuição significativa $(p<0,05)$ no escore médio do FIS na última consulta (Tabela 3 ).

\section{I S CUSSÃO}

No atendimento odontológico a pacientes infantis, uma ferramenta importan-

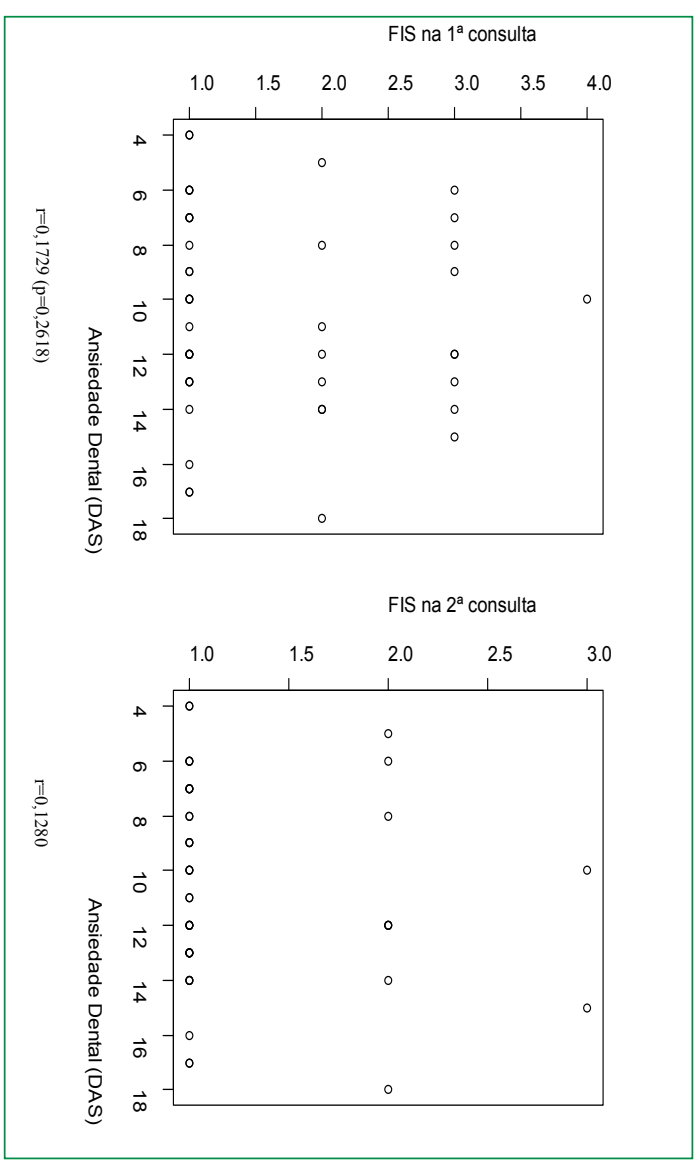

Gráfico 3. Análise de Correlação entre os escores FIS dos pacientes e os escores do DAS dos pais.

te para se lidar com o medo e ansiedade é a desconstrução da imagem negativa do cirurgião-dentista.

É importante a sensibilização dos profissionais da odontologia no sentido de educação continuada sobre a psicologia aplicada para crianças. A colaboração do paciente infantil dentro do consultório está muito condicionada à ausência de medo, ansiedade e dor. Para isto é necessária uma abordagem adequada, a fim de se evitar o desgaste emocional da criança e abandono do tratamento ${ }^{25}$.

Observou-se, nesta pesquisa, uma adequada combinação das Escalas de Ansiedade (Ansiedade Dental de Corah) respondidas por pais e a Escala de Ansiedade Facial (FIS) respondida pelos filhos, permitindo-se visualizar uma apropriada comparação entre essas duas condições. A ansiedade, em um nível moderado e alto, foi prevalente entre os pais participantes deste estudo, porém não foram encontradas associações positivas entre os escores de ansiedade odontológica das crianças e 
seus pais separadamente, corroborando com os estudos ${ }^{19}$.

Apesar de sua larga utilização para avaliação do comportamento infantil, as escalas não podem ser consideradas um instrumento infalível, visto que o examinador pode ser influenciado pelo comportamento da criança, agindo de modo severo, cauteloso ou demasiadamente generoso. Além disso, a ausência de sinais de não colaboração ou de ansiedade não significa que a criança não se sinta apreensiva ou com medo; a criança pode se sentir ansiosa, mas não manifestar externamente esses sinais ${ }^{26}$.

Estudos têm demonstrado que a dor gera certo grau de sofrimento psicológico, podendo motivar também a ansiedade, porém esta pode sofrer variações que dependem de uma série de fatores individuais, pois cada indivíduo tem sua história pessoal, experiências odontológicas anteriores em um contexto socioeconômico e cultural diferentes ${ }^{4,27}$. Essas variáveis não foram verificadas nesta pesquisa, porém considera-se importante essa avaliação em pesquisas futuras.

Autores relataram o comportamento das mães influenciando diretamente $\mathrm{o}$ comportamento dos filhos durante o tratamento odontológico ${ }^{28}$. A atitude dos pais também exerce influência sobre o comportamento dos filhos no consultório odontológico, e pesquisas têm mostrado que crianças ansiosas possuem pais que apresentam maior grau de ansiedade ao tratamento odontológico em comparação com crianças não ansiosas ${ }^{19,29}$. Nesta pesquisa a maioria dos pais apresentou comportamento de moderada à ansiedade exacerbada. Também não houve correlação significativa entre os escores de FIS dos pacientes e as questões do instrumento DAS dos pais nas quatro situações avaliadas nas duas consultas.

Goettms et al..$^{30}$ avaliaram a influência da ansiedade materna relacionada com o comportamento do filho no atendimento odontológico e na saúde bucal, e o estudo mostrou que os filhos de mães ansiosas são mais propensos a apresentar saúde bucal comprometida. Os dados desta pesquisa se contrapõem a esses resultados, já que não houve correlação entre a ansie- dade dos pais e o comportamento ansioso dos filhos quando na cadeira do dentista.

Uma variável normalmente pesquisada para relacionar a ansiedade e o medo da criança diante de tratamentos odontológicos é a experiência prévia com a anestesia. Não há um consenso sobre o assunto. A qualidade dessas experiências parece ser crucial para determinar o grau de ansiedade induzida na criança, podendo tanto exacerbar como atenuar o medo ${ }^{31}$, 32, Essas variáveis não foram estudadas nesta pesquisa, mas sugere-se a inclusão delas em pesquisas futuras que avaliem o medo de crianças em relação ao tratamento odontológico, onde comumente se faz o uso de anestesias locais.

O conhecimento em psicologia infantil apresenta ao cirurgião-dentista as condições de compreender cientificamente os problemas comportamentais que ocorrem rotineiramente em seu consultório e, dessa forma, expõe a maneira mais adequada de solucioná-los ${ }^{33}$. Além de técnicas de modelagem bastante recomendadas nas abordagens em Odontopediatria, o uso de vídeos no atendimento para crianças na primeira infância pode ser uma das ferramentas para a diminuição dos níveis de ansiedade durante as consultas, podendo resultar em comportamentos mais favoráveis durante os procedimentos ${ }^{34}$.

A aplicação de desenhos ao paciente infantil como método de observação do seu grau de ansiedade se mostrou bem eficaz nesta pesquisa, fato este encontrado em outros estudos ${ }^{14,16,17,21 .}$

Pesquisadores analisaram a ansiedade infantil de pacientes e observaram que as crianças na faixa pré-escolar, entre três e seis anos de idade, apresentam mais chances de ter ansiedade na consulta odontológica do que crianças com idade escolar de 7 a 12 anos. Verificaram que crianças a partir de sete anos de idade apresentam maior facilidade em cooperar, já que o período escolar promove proximidades com o convívio diário ${ }^{19}$. Neste estudo a diferença entre nível de ansiedade e a faixa etária menor ou maior não foi observada. Alguns estudos relatam que o aumento da idade amplia a capacidade de raciocínio e compreensão da criança permitindo maior facilidade na adaptação ao
FERREIRA HACM

OLIVEIRA AMG

ANSIEDADE ENTRE CRIANÇAS E SEUS RESPONSÁVEIS PERANTE O ATENDIMENTO ODONTOLÓGICO.
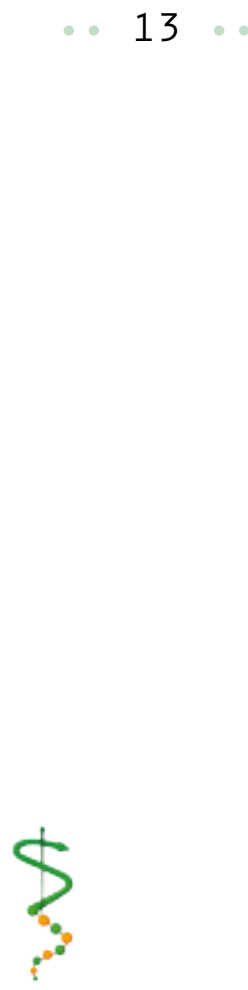

REV, ODONTOL,

UNIV, CID, SÃO PAULO

2016; 29(1): 6-17, JAN - ABR 
FERREIRA HACM :

OLIVEIRA AMG

ANS IEDADE ENTRE CRIANÇAS E SEUS RESPONSÁVEIS

PERANTE O

ATENDIMENTO ODONTOLÓGICO.

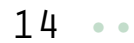

REV. ODONTOL. UNIV, CID, SÃO:

PAULO

$2017 ; 29(1): 6-17$ $J A N-A B R$ tratamento odontológico. Diferentemente, crianças na faixa etária entre cinco a seis anos apresentam um grande medo nesta etapa, sendo que o medo às lesões corporais ou mais leve dano físico é bastante generalizado e difícil de compreender ${ }^{3,33}$. Acredita-se que, neste estudo, uma amostra que contemplasse pacientes adolescentes, com diferenças no nível de ansiedade poderiam ser significantes. Esse fato pode vir a ser um viés na pesquisa, já que crianças maiores podem ter índices de cárie mais elevados, mais tratamentos invasivos, o que contribuiria com o aumento dos escores de ansiedade com a idade ${ }^{35}$.

Uma das questões sobre ansiedade é a existência ou não de diferença entre os sexos. Essa diferença não foi verificada nesta pesquisa, corroborando com os estudos citados em ${ }^{13,19,27}$. Entretanto, nos resultados encontrados nos estudos de Bottan et al. ${ }^{4}$, Sighn et. al. ${ }^{34}$, Lee et. $a^{\beta 6}$, Tickle et al. ${ }^{37}$ as meninas apresentaram maior ansiedade $(p<0,05)$ que os meninos, apresentando escores mais altos de ansiedade. Essa diferença também foi encontrada em estudos sobre ansiedade geral e outros distúrbios psicológicos, contribuindo para reforçar a hipótese de que mulheres são mais ansiosas que os homens ${ }^{38}$. Ao se avaliar de forma geral, observou-se que, das crianças que participaram deste estudo, a maioria apresentou-se ansiosa na primeira consulta, resultados do FIS marcando a carinha triste ou muito triste, diferente da representação do FIS para a última consulta $(p=0,0015)$.

A maior compreensão do mundo infantil e a experiência odontológica podem ser vivenciadas de forma mais prazerosa e educativa aos pacientes infantis, sendo também mais recompensadoras para $\mathrm{O}$ profissional que as pratica ${ }^{22}$. Nesta pesquisa, houve diminuição significativa no escore médio do FIS na última consulta, verificou-se que o acolhimento do paciente pelo profissional, bem como a forma de se comunicar, pode ter facilitado essa melhora significativa do escore de ansiedade da criança. Normalmente, na consulta odontopediátrica a criança é acompanhada pelos pais ou responsável, sendo importante esse contato triplo, pois o profissional nesse momento obtém maior co- nhecimento e informações sobre o comportamento da criança no seu contexto social, podendo assim desenvolver uma técnica de comunicação personalizada para aquele momento e planejar melhor as próximas consultas, promovendo, desse modo, a confiança do paciente infantil.

O profissional deve promover o acoIhimento do paciente ansioso, passando segurança e respeitando a individualidade de cada um, pois, uma vez conhecidas mais profundamente as características de cada criança, pode-se reverter a ansiedade no consultório odontológico para um momento mais tranquilo de consulta, através da sua conquista, suprindo algumas dessas carências afetivas e tornando o momento da consulta odontológica um momento de lazer para as crianças ${ }^{33}$.

A prática odontológica e principalmente a Odontopediatria, não podem desconhecer a prevalência da ansiedade no tratamento dentário. Estratégias para avaliação, prevenção e controle de ansiedade podem ser implementadas para uma meIhor abordagem de crianças e seus pais ${ }^{39}$.

Algumas limitações desta pesquisa, como a amostra reduzida, fizeram com que correlações encontradas previamente na literatura não tenham sido demonstradas de forma contundente. Acredita-se que um maior número de participantes, idades mais elevadas, poderia influenciar em alguns dados, aumentando a tendência de ocorrência de associação ou de diferenças estatisticamente significativas.

Infere-se que o conhecimento do contexto social em que a criança vive é importante para o profissional desenvolver a melhor forma de se comunicar com seu paciente infantil, o que auxiliaria, dessa forma, na diminuição da taxa de ansiedade deste durante o tratamento, diminuindo, portanto, o estresse profissional.

\section{CONCLUSÃO}

Observou-se alto grau de ansiedade dos pais, porém esse fator não interferiu no quadro de ansiedade dos pacientes. A ansiedade na primeira consulta odontológica da criança mostrou-se bastante elevada, diminuindo consideravelmente na última consulta. A comunicação do cirurgião-dentista com seu paciente infantil foi 
importante para desenvolver a confiança do paciente, diminuindo, assim, sua an- siedade perante o tratamento odontológico.
FERREIRA HACM OLIVEIRA AMG

ANSIEDADE ENTRE CRIANÇAS E SEUS RESPONSÁVEIS PERANTE O ATENDIMENTO ODONTOLÓGICO.

\section{REFERÊNCIAS}

1. Guedes-Pinto A. Conduta clínica e psicologia. In: Guedes-Pinto A, editor. Odontopediatria. 8 ed. São Paulo: Edição Ouro; 2010.

2. Barreto R, Pereira G. Farmacoterapia na clínica odontológica. João Pessoa: Editora Universitária (UFPB); 2008.

3. Toledo A, Rocca R. Odontopediatria: fundamentos para a prática clínica. Rio de Janeiro: Medbook; 2012.

4. Bottan E, Lehmkuhl G, Araújo S. Ansiedade no tratamento odontológico: estudo exploratório com crianças e adolescentes de um município de Santa Catarina. RSBO. 2008;5:13-9.

5. Kanegane K, Penha S, Borsatti M, Rocha R. Ansiedade ao tratamento odontológico em atendimento de urgência. Revista de Saúde Pública. 2003;37(2):786-92.

6. Crocombe LA, Broadbent JM, Thomson WM, Brennan DS, Slade GD, Poulton R. Dental visiting trajectory patterns and their antecedents. Journal of public health dentistry. 2011;71(1):2331.

7. van Maanen EJ, van Dinter N, Versloot J, Veerkamp JS. [Fear of dental treatment among children. Influence of experience and psychological functioning]. Nederlands tijdschrift voor tandheelkunde. 2009;116(1):3-8.

8. Chhabra N, Chhabra A, Walia G. Prevalence of dental anxiety and fear among five to ten year old children: a behaviour based cross sectional study. Minerva stomatologica. 2012;61(3):83-9.
9. Ferreira MA, Manso MC, Gavinha S. Ansiedade e fobia dentária: avaliação psicométrica num estudo transversal. Revista Portuguesa de Estomatologia, Medicina Dentária e Cirurgia Maxilofacial. 2008;49(2):77-86.

10. Coura LR, Zöllner N, Zöllner NA, Laureano da Rosa LC, Ferreira de Medeiros JM. Estudo comparativo de dois procedimentos de aplicação de anestesia local intraoral. Revista Portuguesa de Estomatología, Medicina Dentária e Cirugia Maxilofacial. 2011;52(2):70-6.

11. Themessl-Huber M, Freeman R, Humphris G, MacGillivray S, Terzi N. Empirical evidence of the relationship between parental and child dental fear: a structured review and meta-analysis. International Journal of Paediatric Dentistry. 2010;20(2):83-101.

12. Ramos-Jorge ML, Marques LS, Pavia SM, Serra-Negra JM, Pordeus IA. Predictive factors for child behaviour in the dental environment. European archives of paediatric dentistry : official journal of the European Academy of Paediatric Dentistry. 2006;7(4):253-7.

13. Ribas TA, Guimarães VP, Losso EM. Avaliação da ansiedade odontológica de crianças submetidas ao tratamento odontológico. Arquivos em Odontologia. 2016;42(3).

14. Aminabadi NA, Ghoreishizadeh A, Ghoreishizadeh M, Oskouei SG. Can drawing be considered a projective measure for children's distress in paediatric dentistry? International journal of paediatric dentistry. 2011;21(1):112. 
FERREIRA HACM :

OLIVEIRA AMG

ANS IEDADE ENTRE CRIANÇAS E SEUS RESPONSÁVEIS

PERANTE O

ATENDIMENTO ODONTOLÓGICO.

\section{ISSN 1983-5183}

15. Mochidome F. Avaliação do medo infantil à assistência odontológica através de um método projetivo modificado [Tese]. Universidade Estadual Paulista aú́lio de Mesquita Filho" 2006.

16. Marques KBG, Gradvohl MPB, Maia MCG. Medo e ansiedade prévios à consulta odontológica em crianças do município de Acaraú-CE. Revista Brasileira em Promoção da Saúde. 2010;23(4).

17. Aminabadi NA, Puralibaba F, Erfanparast $L$, Najafpour E, Jamali $Z$, Adhami SE. Impact of temperament on child behavior in the dental setting. Journal of dental research, dental clinics, dental prospects. 2011;5(4):119-22.

18. Silva Menetti FC, Santos Maldonado J, Santos Martins G, Tucunduva MJ. Avaliação da intensidade de dor em pacientes submetidos a tratamentos odontológicos. Science in Health. 2011;2(3):163-9.

19. Oliveira MF, Moraes MVM, Evaristo PC. Avaliação da Ansiedade dos Pais e Crianças frente ao Tratamento Odontológico. Pesquisa Brasileira em Odontopediatria e Clínica Integrada. 2012;12(4).

20. Versloot J, Veerkamp J, Hoogstraten J. Dental anxiety and psychological functioning in children: its relationship with behaviour during treatment. European archives of paediatric dentistry : official journal of the European Academy of Paediatric Dentistry. 2008;9 Suppl 1:36-40.

21. Melo RB, Lima FC, Moura GM, Silva PG, Gondim JO, Moreira-Neto JJ. Avaliação da relação entre procedimentos odontológicos e comportamento infantil. ROBRAC. 2015;23(68).

22. Pereira VZ, Barreto RC, Pereira GAS, Cavalcanti HRBB. Avaliação dos níveis de ansiedade em pacientes submetidos ao tratamento odontológico. Revista Brasileira de Ciências da Saúde. 2013;17(1):55-64.
23. Buchanan $\mathrm{H}$, Niven $\mathrm{N}$. Validation of a Facial Image Scale to assess child dental anxiety. International journal of paediatric dentistry. 2002;12(1):47-52.

24. Corah NL, Gale EN, Illig SJ. Assessment of a dental anxiety scale. Journal of the American Dental Association (1939). 1978;97(5):816-9.

25. Andrade DSPD, Minhoto TB, Campos FdAT, Gomes MC, Granville-Garcia AF, Ferrreira JMS. Percepção infantil através de desenhos e caracterização verbal sobre o cirurgião-dentista. Arquivos em Odontologia. 2013;49(4):184-90.

26. Cardoso CL, Loureiro SR. Estresse e comportamento de colaboração em face do tratamento odontopediátrico. Psicologia em Estudo. 2008;13(1):13341.

27. Oliveira MMT, Colares V. The relationship between dental anxiety and dental pain in children aged 18 to 59 months: a study in Recife, Pernambuco State, Brazil. Cadernos de Saúde Pública. 2009;25(4):743-50.

28. Aminabadi NA, Pourkazemi M, Babapour J, Oskouei SG. The impact of maternal emotional intelligence and parenting style on child anxiety and behavior in the dental setting. Medicina oral, patologia oral y cirugia bucal. 2012;17(6):e1089-95.

29. Seran N, Demopoulos C, Mobley C, Ditmyer M. Parenting style and oral health status. Open Journal of Pediatrics 2013(3):188-94.

30. Goettems ML, Ardenghi TM, Demarco FF, Romano AR, Torriani DD. Children's use of dental services: influence of maternal dental anxiety, attendance pattern, and perception of children's quality of life. Community dentistry and oral epidemiology. 2012;40(5):451-8.

31. Moro ET, Módolo NSP. Ansiedade, a criança e os pais. Revista Brasileira de Anestesiologia. 2004;54(5):728-38.
REV, ODONTOL UNIV. CID, SÃO PAULO JAN - ABR 
32. Davidovich E, Wated A, Shapira J, Ram D. The influence of location of local anesthesia and complexity/duration of restorative treatment on children's behavior during dental treatment. Pediatric dentistry. 2013;35(4):333-6.

33. Góes MPS, Domingues MC, Couto GBL, Barreira AK. Ansiedade, medo e sinais vitais dos pacientes infantis. Odontologia Clínico-Científica (Online). 2010;9(1):39-44.

34. Singh KA, Moraes ABAd, Bovi Ambrosano GM. Medo, ansiedade e controle relacionados ao tratamento odontológico. Pesquisa Odontológica Brasileira. 2000;14(2):131-6.

35. Lima, RL, Macedo A, Duarte D, Sant'Anna G. Avaliação de parâmetros preditores de ansiedade em crianças de três a cinco anos usando vídeos como instrumento facilitador no tratamento odontopediátrico. Rev Bras Pesq Saúde. 2013;15(1):25-32.
36. Lee CY, Chang YY, Huang ST. Prevalence of dental anxiety among 5to 8-year-old Taiwanese children. Journal of public health dentistry. 2007;67(1):36-41.

37. Tickle M, Jones C, Buchannan K, Milsom KM, Blinkhorn AS, Humphris GM. A prospective study of dental anxiety in a cohort of children followed from 5 to 9 years of age. International journal of paediatric dentistry. 2009; 19(4):225-32.

38. Klingberg G, Broberg AG. Dental fear/ anxiety and dental behaviour management problems in children and adolescents: a review of prevalence and concomitant psychological factors. International journal of paediatric dentistry. 2007;17(6):391-406.

39. Aminabadi NA, Adhami ZE, Oskouei SG, Najafpour E, Jamali Z. Emotional intelligence subscales: are they correlated with child anxiety and behavior in the dental setting? The Journal of clinical pediatric dentistry. 2013;38(1):61-6.

Recebido em 10/10/2016

Aceito em 01/12/2016
FERREIRA HACM

OLIVEIRA AMG

ANSIEDADE ENTRE CRIANÇAS E SEUS RESPONSÁVEIS PERANTE O ATENDIMENTO ODONTOLÓGICO.

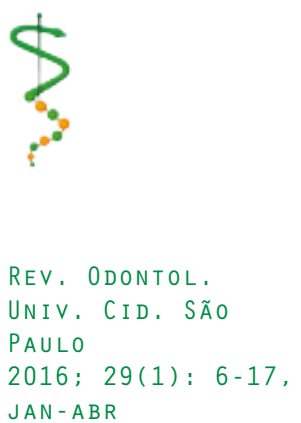

